cemoti $\begin{aligned} & \text { Cahiers d'études sur la Méditerranée } \\ & \text { orientale et le monde turco-iranien }\end{aligned}$

19 | 1995

Laïcité(s) en France et en Turquie

\title{
Yilmaz Güney : le cinéaste révolté
}

\section{Schofield CORYELL}

\section{(2) OpenEdition}

\section{Journals}

Édition électronique

URL : http://journals.openedition.org/cemoti/1709

ISSN : 1777-5396

Éditeur

AFEMOTI

Édition imprimée

Date de publication : 1 janvier 1995

ISSN : 0764-9878

\section{Référence électronique}

Schofield CORYELL, "Yilmaz Güney: le cinéaste révolté 》. Cahiers d'études sur la Méditerranée orientale et le monde turco-iranien [En ligne], 19 | 1995, mis en ligne le 14 mai 2006, consulté le 07 septembre 2020. URL : http://journals.openedition.org/cemoti/1709

Ce document a été généré automatiquement le 7 septembre 2020

Tous droits réservés 


\section{Yilmaz Güney : le cinéaste révolté}

\section{Schofield CORYELL}

1 Il y a dix ans, Yilmaz Güney, le grand cinéaste kurde né en Turquie, est mort en exil à Paris, à l'âge de 47 ans. Il laisse derrière lui une vaste oeuvre cinématographique, témoignage poignant de la misère des paysans de son pays d'origine, mais aussi de leurs aspirations à la justice et au bonheur.

2 Güney était l'exemple par excellence d'un artiste d'un certain type - l'artiste dont la vie et les convictions ne font qu'un avec son oeuvre. Son travail et sa vie sont aux antipodes de "l'objectivité" pronée par certains à une époque qui serait celle de la mort pas seulement de l'idéologie mais de l'histoire elle-même.

3 La filmographie de Güney aurait sans doute été plus imposante encore si son travail de cinéaste n'avait été trop souvent interrompu par de longs séjours en prison. Car cet artiste a chèrement payé son engagement aux côtés de toutes les victimes de ce monde, de son monde. Ses films traitent de la vie quotidienne dans les campagnes et de l'oppression qui s'abat continuellement sur les paysans qui tirent péniblement une maigre subsistance de la terre.

4 Parmi les films de Güney se trouvent des titres aussi connus que "Yol" (Palme d'Or au Festival de Cannes, 1982), histoire de détenus qui retournent dans leur village pour une courte permission, ou "Le Troupeau", qui décrit l'écart entre deux mondes, celui des campagnes où vit encore la moitié de la population et les grandes villes modernes, avec leurs gratte-ciels et leurs bidonvilles.

5 A ces films s'ajoutent d'autres - par exemple, "L'Espoir", "Elégie", "Les Malheureux" qui dramatisent des thèmes constants d'actualité et d'histoire contemporaine : l'exode rural, entraînant le dépeuplement des campagnes et le gonflement malsain des villes ; l'inégale distribution des revenus, provoquant les souffrances matérielles et morales du plus grand nombre pour le seul bénéfice d'une poignée de nantis ; le mécontentement sourd des paysans et ouvriers agricoles face à la puissance arbitraire et brutale des propriétaires terriens.

6 Dans un des trois romans que Güney a écrits pendant ses années de prison, il fait dire à un vieux paysan profondément religieux qui assiste aux funérailles de l'un des siens, écrasé par le fardeau de la vie : "La terre nous nourrit lorsque nous mourons et nous 
tyrannise lorsque nous sommes en vie. Elle nourrit certains de leur vivant, mais nous seulement lorsque nous sommes morts".

7 Mais ces paroles de résignation et de tristesse ne correspondent pas tout à fait à l'attitude de Güney lui-même, dont les opinions démocratiques et populistes ont souvent irrité les autorités. C'est pourquoi le cinéaste a été plusieurs fois condamné et incarcéré pour des idées jugées intolérables en haut lieu. Les dates de ses arrestations successives marquent des moments intenses de sa vie d'artiste et d'homme, mais aussi, et tout autant, des moments particulièrement troublés de l'histoire politique de la Turquie - moments où les intellectuels critiques et insoumis sont choisis régulièrement comme cibles de la répression et boucs émissaires.

8 Ainsi, Yilmaz a été arrêté et détenu pendant 18 mois en 1961, au lendemain d'un coup d'Etat militaire qui avait soulevé beaucoup d'espoirs de changement démocratique espoirs vite déçus par la tournure des événements. L'accusation contre lui ?:"Propagande communiste". Les preuves ? : Il avait mis les paroles suivantes dans la bouche d'une petite paysanne, personnage d'une de ses premières nouvelles publiées dans une revue d'Istanbul : "Si tous les gens étaient égaux, le monde serait un paradis !"

9 Güney a été emprisonné neuf ans plus tard, en 1972, au moment d'un grand sursaut du mouvement ouvrier auquel a répondu une pression intense des militaires, sous forme d'un semi-putsch faisant du régime le captif de l'armée. Le cinéaste était accusé d'avoir "aidé et hébergé des révolutionnaires armés" - il était soupçonné d'entretenir des relations avec le groupe d'extrême-gauche, Dev Genç, dont les actions violentes défrayaient à l'époque la chronique. Güney s'est donc retrouvé pendant deux ans derrière les barreaux.

10 Libéré en 1974 à la faveur d'une amnistie générale proclamée sous le gouvernement social-démocrate de Bülent Ecevit, il n'a pu jouir que d'un bref moment de liberté car il était arrêté de nouveau trois mois plus tard, accusé cette fois d'avoir tué un juge au cours d'une bagarre. L'incident a eu lieu dans le bistro d'un village proche d'Adana où Güney dirigeait le tournage d'un film, "Inquiétude", sur les souffrances et épreuves des travailleurs saisonniers des champs de coton. Il s'agissait de toute évidence d'une provocation et il n'a jamais été formellement preuve que Güney avait effectivement tiré la balle fatale.

11 Cet épisode tragique a valu à Güney une condamnation à 18 ans de prison.

12 Etant donné les liens étroits entre les convictions de Güney et le message implicite ou explicite de son oeuvre, on voit qu'il a payé un prix élevé pour ses idées et ses paroles critiques à l'égard d'un Etat dont la tolérance politique n'est pas la principale vertu. Mais, s'il s'était fait des ennemis puissants et haut placés dans la société turque, le sens de l'engagement et la sincérité passionée de Güney lui ont valu en même temps une gamme étendue d'amitiés politiques et personnelles.

13 Il n'est donc pas surprenant que plusieurs milliers de personnes aient assisté aux funérailles de Güney au cimetière du Père Lachaise, le 13 septembre 1984 - des Turcs et des Kurdes de toutes conditions, exilés à Paris comme lui, mais aussi beaucoup d'amis français et étrangers : artistes, hommes et femmes politiques (Jack Lang, ministre de la Culture à l'époque, était présent, ainsi que Madame Danielle Mitterrand).

14 Parmi les nombreuses couronnes déposées sur la tombe de Güney se trouvaient notamment celles des diverses organisations de la gauche turque, des Kurdes de Turquie et d'Iran, des "Communistes de Grèce", des "Anciens combattants arméniens 
en France", des travailleurs de la confection (CFDT) et de plusieurs syndicats de l'industrie du cinéma. L'oraison funèbre a été prononcée par un universitaire turc, Server Tanilli - paralysé au-dessous de la ceinture par la balle d'un criminel qui a tiré sur lui à Istanbul. En exil, il enseigne à l'Université de Strasbourg.

15 Telle a été la mort de Güney, mais quelles étaient ses origines, d'où venait-il ? Ses films - ainsi que ses romans - traitent presque tous de la vie qu'il a connue en tant que fils d'une famille de paysans pauvres, dans la région d'Adana. C'est le centre du "pays du coton" où quelques grands aghas, propriétaires terriens, règnent en maîtres sur une population qui arrive à grand peine à survivre.

16 La mère de Güney, Güllü, venait d'une riche famille kurde qui, obligée de fuir la région de l'Est pendant la Première guerre Mondiale, s'était installée aux environs d'Adana où Güllü avait épousé un ouvrier agricole. Yilmaz s'est souvenu toute sa vie des chansons mélodieuses que sa mère lui chantait lorsqu'il était enfant dans sa langue maternelle, le kurde.

17 Le foyer familial était heureux jusqu'au jour où le père de Yilmaz, après avoir pris une deuxième femme, s'était mis à battre régulièrement Güllü qui devait souvent se sauver avec ses enfants.

18 Il n'est pas irréaliste de supposer que ces circonstances de la vie familiale ont profondément marqué la personnalité de Güney, le rendant particulièrement sensible aux problèmes des Kurdes et des ouvriers agricoles en général, tout en lui donnant un sens aigu de l'injustice ainsi qu'une haine féroce de l'oppression sous toutes ses formes. Ce sont effectivement les caractéristiques de toute son oeuvre cinématographique et littéraire ultérieure.

19 Si la mère de Yilmaz était analphabète, son père avait pu apprendre à lire et à écrire pendant son service militaire. Bien que n'ayant pas eu d'autre école, il était devenu, grâce à son "bon sens" naturel, une sorte de "sage" respecté et consulté par tout le village. Mais il avait d'autres ambitions pour son fils : "il voulait que je fasse des études afin de pouvoir sortir des horizons limités du milieu rural et faire mon chemin dans le monde, devenir un fonctionnaire, par exemple, et porter - pourquoi par ? - un costume et une cravate" ${ }^{\prime 1}$.

20 Dans ce contexte, l'école - un bâtiment blanc aux tuiles rouges, entouré d'arbres" revêtait pour le garçon et ses parents une importance quasi-mystique, la clé magique d'un bonheur futur : "son espoir, son avenir, sa vie", écrit Güney dans son roman largement autobiographique, "Les Champs de Yuréghir". C'est cette espérance obstinée qui justifiait en effet les trois heures de marche que faisait chaque jour le petit Güney pour gagner l'école du village voisin où il cherchait à acquérir les éléments rudimentaires - et indispensables - d'une éducation de base.

21 Les parents de Güney n'auraient sans doute pu imaginer la voie que leur fils allait suivre. Celui-ci s'est trouvé plongé -d'une façon inattendue - dans le monde du cinéma. A l'âge de vingt ans, il a été embauché par une compagnie de distribution de films. Son travail consistait à aller de village en village pour projeter des films à la population locale. "A force de voir et de revoir le même film, je finissais - se rappelait-il - par comprendre le comment et le pourquoi de tel ou tel plan. C'était une initiation formidable".

22 Sa chance - et le commencement d'une longue et intense carrière - est venue un an plus tard, en 1958. Son inculpation à la suite de la publication de sa première nouvelle 
lui a valu d'être licencié par la compagnie de distribution qui l'employait à ce moment là. Privé ainsi de son salaire, modeste certes mais régulier, et faisant face à une période pénible de chômage, Güney a été sauvé par l'intervention de deux compatriotes célèbres : le romancier Yachar Kemal et le cinéaste Atif Yilmaz. Ils préparaient alors un film "Les enfants de la patrie" sur la guerre de l'indépendance et - par solidarité avec un jeune artiste en difficulté - ils ont proposé à Güney de collaborer à l'élaboration du scénario. Son apport leur a paru si intéressant qu'ils ont décidé de prendre Güney comme deuxième assistant. Enfin, l'absence d'un des acteurs au moment du tournage lui a donné l'occasion de jouer le rôle mineur d'un jeune paysan engagé dans la résistance nationale contre l'occupant.

Ainsi débuta la longue et brillante carrière cinématographique du jeune artiste inconnu qu'était alors Güney. Ce dernier fut étonné quand Atif Yilmaz le choisit pour le rôle principal de son film suivant, "le Cerf Rouge", car Güney savait bien qu'il n'avait pas le physique d'un "jeune premier". Atif l'a pourtant assuré : "Tu es le type même du jeune paysan - et en plus tu sais monter à cheval, manier un fusil, grimper aux arbres. Tu es le chasseur de cerfs idéal".

24 A partir de ce moment-là, Güney est devenu, comme il le disait lui-même, un "fanatique du cinéma", ouvert à tous les genres, à toutes les influences. Il suivait avec passion les vedettes américaines comme George Raft, Humphrey Bogart, Marlon Brando et appréciait les techniques et les conceptions des réalisateurs tels que Kazan, Bergman et surtout les Italiens Visconti, Vittorio de Sica, Rossellini, etc. "J'apprenais quelque chose de chacun d'eux, je cherchais à assimiler les techniques et les conceptions les plus diverses", disait-il.

25 Pendant qu'il était en prison dans les années 60, Güney avait bien réfléchi à sa future carrière, en tenant compte de la situation politique et personnelle où il se trouvait. $\mathrm{A}$ l'époque, il avait établi toute une stratégie pour sa réussite : "Mon ambition, disait-il, était de devenir réalisateur, mais je savais qu'après ma condamnation ce ne serait possible qu'à une seule condition : devenir d'abord l'acteur le plus populaire de Turquie. J'arriverais ainsi à mes fins par des chemins détournés".

26 Güney a gagné son pari audacieux car il est effectivement devenu l'acteur le plus populaire du pays, jouant les rôles principaux dans film après film d'action et d'aventures. Représentant le plus souvent la force morale et le courage des "petites gens" contre les tracasseries et les manoeuvres des "gros" et des puissants, Güney était devenu un véritable mythe populaire sous le sobriquet du "Roi laid", titre d'un de ses films de l'époque. Dans les années 60-70, des posters à son effigie s'étalaient chez les marchands de journaux et sur les trottoirs d'Istanbul et d'Ankara.

27 Enfin, grâce à ses gains d'acteur, Güney put fonder sa propre société de production et faire ses débuts de réalisateur en 1968, avec un film intitulé "Seyyit Han". Son premier succès international a été "L'Espoir" (Umut), qui a remporté l'Ours d'Or à Berlin en 1970. Ce film autobiographique est fondé sur les observations de Güney adolescent sur sa famille et son milieu social. En 1971, il réalisait "Elégie" (Agit) qui raconte la vie pleine de risques des contrebandiers d'Anatolie du sud-est.

28 Par la suite, c'est surtout depuis sa cellule que Güney a continué à écrire des scénari et à diriger des films qui ont confirmé sa notoriété à l'étranger aussi bien qu'en Turquie même. "Inquiétude" (Endise), le film qu'il réalisait au moment de son incarcération en 1974, a été tourné sur ses indications détaillées par son assistant Serif Gören, également 
le réalisateur de "Yol". "Le Troupeau" (Sürü), sorti en 1978, a été signé par un autre de ses assistants, Zeki Ökten.

29 En prison, Güney n'a jamais cessé d'insister sur les droits humains élémentaires des détenus, et cela sans faire de distinction entre les prisonniers politiques et les autres. Dans chacune de ses prisons - car il était souvent transféré de l'une à l'autre, tant les autorités craignaient son influence - il organisait les détenus pour imposer certaines améliorations et tenter d'empêcher la torture.

30 Güney nous a laissé un précieux document, un texte intéressant et percutant sur le sens de son expérience en prison, sur la psychologie aussi bien des prisonniers que des inquisiteurs et tortionnaires. Il s'agit des "Lettres de la Prison de Selimiye", texte adapté et mis en scène à Paris en 1986 par Marianik Révillon sous le titre "Lettres d'un inculpé". Güney y fait parler, par exemple, "Le Colonel", chef des tortionnaires, qui exprime, avec une franchise brutale, sa "philosophie" policière et simpliste : "Ici, il n'y a pas de Constitution, il n'y a pas de Droit, il n'y a pas de machin universel des Droits de l'homme. Ici, il n'y a que les lois de la guerre".

31 Mais ces lettres de prison de Güney sont aussi et surtout autant de chants d'amour et de lutte. Elles sont un lien vivant non seulement avec sa femme, à qui il s'adresse, mais avec le monde entier, avec les révolutionnaires et opprimés de tous les pays : "Mes lettres seront, du moins je le crois, une goutte d'eau vive dans la mare des colères silencieuses. Que d'autres lettres répondent aux miennes, comme les miennes répondent à d'autres qui les ont précédées. D'Espagne, d'Argentine, du Chili, du Brésil, d'Asie, d'Adana, de Diyarbakir et de Sinop ... Des prisons d'Istanbul, du Pérou, de Bolivie et de partout ... Par milliers, par millions, lettres de passion, de résistance ..."

32 A cet esprit internationaliste, à cette conscience aiguë de l'existence d'une opposition planétaire à l'impérialisme, se joint une passion insatiable pour toutes les manifestations de la vie quotidienne au dehors des murs de la prison : "Ma belle enfant, dit-il, je veux te parler de ce qui est beau, de ce qui est espoir, de ce qui est lumière. Je veux te parler des prés, des sources d'été, de la mer, de la beauté des amitiés. Ma belle enfant, nous purifierons tous les humains dans l'océan de mon coeur, nous les rendrons heureux".

33 Après le coup d'Etat militaire du 12 septembre 1980, lorsque l'armée a pris les affaires du pays directement en main, la situation de Güney dans la prison a vite empiré au point où il lui devenait impossible de poursuivre à l'intérieur des murs son travail de création ou ses activités d'organisation des détenus.

34 Un an après le coup d'Etat - en octobre 1981 - Güney a profité d'une brève permission pour prendre le chemin de l'exil, grâce à la complicité de ses nombreux amis et admirateurs en Turquie et en Europe.

35 Avec l'aide et le soutien de Melina Mercouri, alors ministre de la Culture en Grèce, qui est intervenue auprès du ministre de l'Intérieur français, Gaston Defferre, Güney a pu gagner la France et y trouver asile, juste à temps pour assister au Festival de Cannes en 1982 et y recevoir la Palme d'Or pour "Yol" dont il avait pu lui-même terminer le montage.

36 Installé à Paris avec sa femme et ses deux enfants - une fille de 18 ans et un garçon de 11 ans - qui avaient quitté la Turquie peu de temps avant lui, Güney y a réalisé "Le Mur", un film aussi dur que véridique sur les tortures infligées aux adolescents dans les prisons turques. 
37 Ce dernier film n'avait pas l'impact artistique de certaines de ses autres oeuvres, mais comme le disait son compatriote, le poète Nazim Hikmet - "C'est un dur métier que l'exil", et le cinéaste révolté n'a sans doute pas trouvé en France, et dans la banlieue parisienne où il tournait "Le Mur", le contact dont il avait besoin avec son équipe familière et ses amis, ni les paysages de son pays d'origine. Entouré de sympathie et malgré tous les moyens mis à sa disposition, il se trouvait quand même isolé de l'essentiel, des sources de son inspiration. En outre, il était déjà rongé par la maladie le cancer qui a fini par l'emporter en 1984.

\section{NOTES}

1. Les citations directes de Güney non tirées de ses oeuvres viennent d'un entretien que l'auteur a eu avec lui en 1983. 